\title{
DEPRESSION SYMPTOM TRANSITIONS IN OLDER AGE: A MULTI-STATE STUDY
}

\author{
Alejandro de la Torre-Luque, Javier de la Fuente, Jose Luis Ayuso-Mateos. \\ Centre for Biomedical Research in Mental Health CIBERSAM, Spain. Universidad Autonoma de Madrid, Spain.
}

Contact: joseluis.ayuso@uam.es

Background and aims

\begin{abstract}
Depression is very frequent in older age [1,2]. Almost one in four adults may experience a depression disorder episode in late life Up to $10 \%$ of older adults may experience an unremitting and chronic course of depressive symptoms. Finally, recurrent depression prevalence is between $25-40 \%$ in late life.

Depression seems to evolve in older age, dynamically transitioning throughout discrete disorder statuses Little is know about the temporal dynamics of depressive symptom episodes and concomitants.

This study aimed. 1 ) to analyse the temporal dynamics of clinically relevant depression symptoms in older population and 2 ) to examine the influence of risk factors on symptom episode emergence and persistence.
\end{abstract}

\section{Methods}

Data from the Ageing Trajectories of Health - Longitudinal Opportunities and Synergies (ATHLOS) project (http://athlosproject.eu/), an ambitious initiative of harmonisation of longitudinal studies on ageing, was used. [3]

More concretely, data were taken from six longitudinal studies: English Longitudinal Study of Ageing (ELSA); Health and Retirement Study (HRS); Japanese Study of Aging and Retirement (JSTAR); Korean Longitudinal Study of Aging (KLOSA); Mexican Health and Aging Study (MHAS); and the Survey of Health, Ageing and Retirement in Europe (SHARE).

A sample of 41,362 older adults $(54.61 \%$ women; $M=75.30, S D=6.20)$ were followed over an 18-year period.

A multi-state model was fitted considering three depressive symptom episode statuses: no depression, new episode of depressive symptoms (symptom scores over the cut-off point of clinical meaningfulness) and episode persistence (two consecutive assessment occasions with symptoms over the cut-off point of clinical meaningfulness).

A hierarchical mixed-effect multinomial regression was conducted considering 4-transition statuses (no depression in two consecutive waves; new episode emergence; persistence; and remission). Predictors at the individual (sociodemographic and health-related) and cohort level (interaction age ${ }^{*}$ birth year) were added. The level factor was the interaction wave*country.

Results

- Over $5.30 \%$ of individuals showed at least one episode of symptoms in older age (Figure 1) with a significant probability of moving to a persistent symptom status (transition probability $=0.27$ )

- Episode persistence became evident in $9.86 \%$ of episode status transitions.

- Episode recurrence probability (i.e., transition to other symptom episode having had a previous one) was quite high (transition probability $=0.33$ ).

- Individual risk factors were associated with transitions between symptoms emergence and persistence (especially, disability, health status and widowhood). The strongest associations were found for loneliness (OR= 17.76 for symptom emergence, and 5.93 for persistence).

- The cohort effect was also evident but very close-to- 1 coefficients were found.
Figure 1. Symptom episode prevalence over the follow-up.

Solid line = observed prevalence rate. Dashed line = predicted prevalence rate.

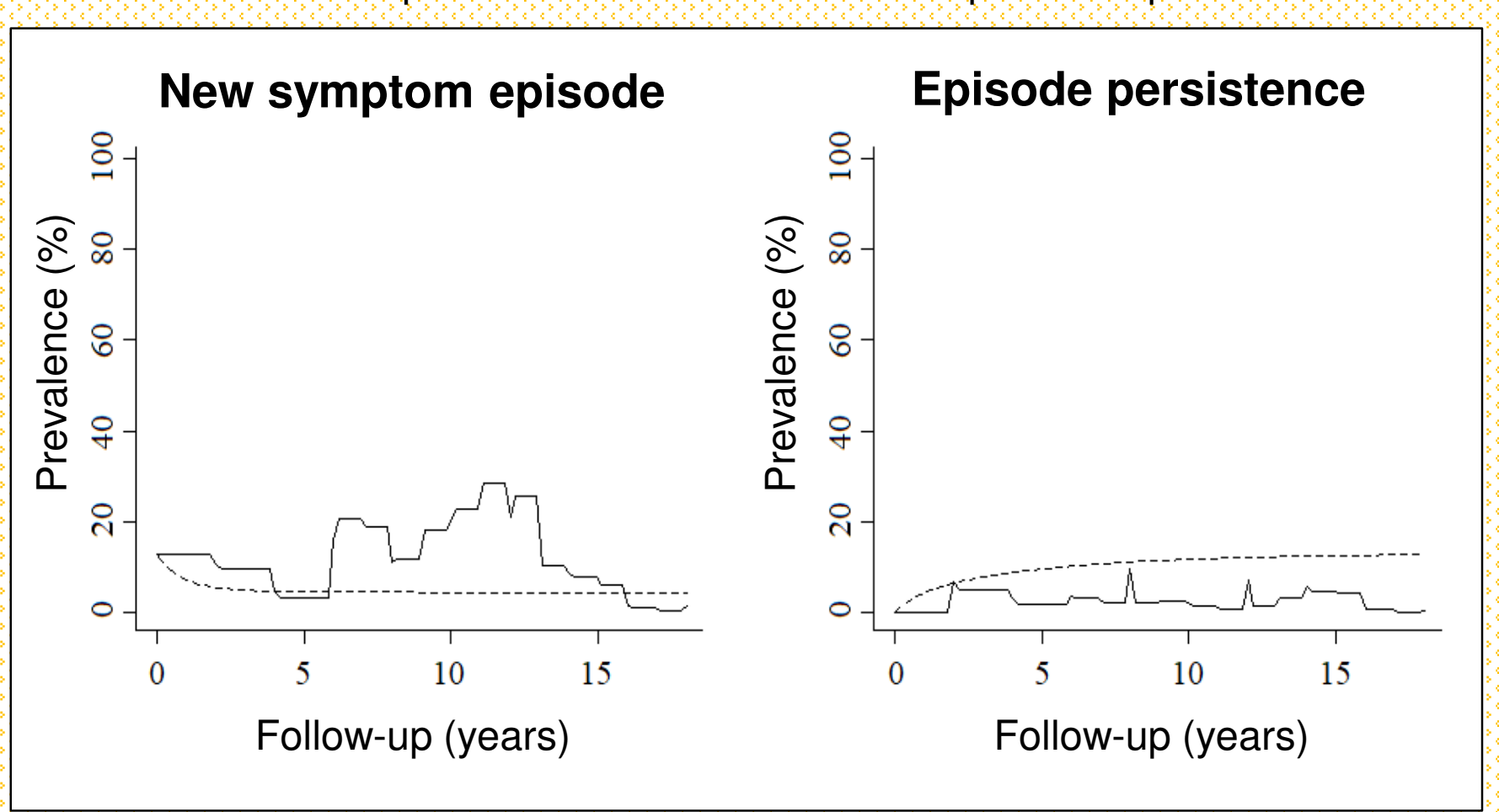

\section{Conclusions}

The probability of manifesting clinically relevant depression symptom episodes raised while ageing, mainly due to increasing episode persistence and recurrence. The influence of individual factors on new episode emergence and persistence was evident in this study, with loneliness being a highly relevant factor. Major efforts should be made to develop tailored action plans in tackling late-life depression and prevent depression from becoming chronic.

References: 1. Rothermund K, et al doi: 10 1037/0882-7974.18.1.80;2. Whiteford HA, et al doi: 10 1017/S0033291712001717,3. Sanchez-Niubo A, et al. doi:10.1093/ije/dyz077 